\title{
Assessment of clinical trial participant patient satisfaction: a call to action
}

\author{
Bethann Mangel Pflugeisen*, Stacie Rebar, Anne Reedy, Roslyn Pierce and Paul J. Amoroso
}

\begin{abstract}
Background: As patient satisfaction scores become increasingly relevant in today's health care market, we sought to evaluate satisfaction of the unique subset of patients enrolling in clinical trials in a research facility embedded within a community hospital system.

Methods: We developed and deployed a patient satisfaction survey tailored to clinical trial patients who consented to and/or completed a clinical trial in our research institute in the prior year. The survey was distributed to 222 patients. Likert scale responses were analyzed using top box and percentile rank procedures. Correlation analysis was used to evaluate associations between the clinical trial experience and intent to return to our system for routine care.
\end{abstract}

Results: Ninety surveys were returned in the 6 months following the mailing for a $41 \%$ response rate; the bulk of these $(N=81)$ were returned within 6 weeks of the mailing. The questions with the highest ranking responses were related to interactions with staff (84th percentile or higher). Fifty-one point one percent of patients (64th percentile) strongly agreed that they would seek future care in our system. Patient intent to return to the provider seen during the clinical trial was most highly correlated with intent to seek future care within our system $(r=0.54, p<0.0001)$. Reasons cited for clinical trial enrollment were generally altruistic.

Conclusions: Querying this special patient population is feasible and yields valuable insight into their experience with healthcare system-based clinical trials and the relationship between clinical trial participation and perception of the healthcare system as a desirable resource for routine medical care. We argue that this work is invaluable to the research community and submit a call to action to our peers to begin systematic evaluation of clinical trial patient satisfaction.

\section{Background}

Patient satisfaction is an increasingly important measure in health care delivery. In the United States, commercial surveys, such as the Hospital Consumer Assessment of Healthcare Providers and Systems (HCAHPS) survey [1], are now conducted on a national scale and used routinely to satisfy internal and external demands for this information. In October 2012, the Centers for Medicare and Medicaid Services began to tie hospital reimbursements to standardized HCAHPS scores [2]. As the health care market becomes increasingly competitive and consumer-driven, patient satisfaction scores have the potential to influence consumer decisions about the utilization of health care resources, and studies have

* Correspondence: bethann.pflugeisen@multicare.org

MultiCare Institute for Research and Innovation, 314 Martin Luther King Jr. Way, Suite 402, Tacoma, WA 98405, USA demonstrated positive associations between overall patient satisfaction and clinical outcomes [3, 4]. Standardization of patient satisfaction scores empowers health care consumers and leaders alike to evaluate and compare a consistent measure of the patient experience between institutions. Finally, it has been shown that patients who are "merely satisfied" with their health care experience are likely to seek future care elsewhere [5], an important finding for competing health care systems that are at risk of losing customers in response to low satisfaction.

Health care organizations with embedded research programs likely experience substantial crossover between research patients and patients seeking routine care; research may attract new patients to the system and the system can engage existing patients in research. Overall patient satisfaction has been shown to correlate most highly with communication with nurses [6], and research patients in 
an embedded research division interact with an additional team of study staff, often nurses and/or certified clinical research coordinators/assistants, who represent the larger organization. While the satisfaction of patients seeking routine care is now formally measured within most large health care systems [7], and the motivation of research patients for participating in clinical trials has been welldescribed [8-12], there is a dearth of information regarding the satisfaction of research patients with their clinical trial experience or the relationship between research patient satisfaction and use of the host institution for routine medical care. Notably, the 3rd International Clinical Trials Methodology Conference held in Glasgow in late 2015 included two posters addressing the patient experience of clinical trial participants. One systematic literature review (1995-2014) identified only five research patient experience measures [13], while a second reported results of a survey conducted with 44 clinical trial participants [14]. This suggests that while an international discussion about patient satisfaction is underway, it remains nascent.

Several unique aspects of the research patient experience, such as informed consent and patient incentives for study enrollment, are not part of routine care and thus cannot be evaluated by standardized patient experience surveys designed to assess the provision of nonresearch health care services. Two substantial gaps are created by the absence of a standardized and widely used survey explicitly designed to assess the unique patient experience of clinical trial participants: first, the research community operates in the absence of benchmarks by which to identify programmatic strengths/weaknesses and upon which to base improvements; second, the patient voice is lost.

We sought to develop an independent questionnaire that would (1) evaluate the satisfaction of patients participating in clinical trials within our research institute, (2) establish internal, measureable benchmarks to allow ongoing evaluation of service to our clinical trial participants, and (3) identify existing associations between patient engagement in clinical trials and use of our health care system for nonresearch medical care. Perhaps most importantly, however, this work is a call to action for the broader research community: it is time that we engage in a national/international conversation about the importance of measuring the experience of clinical trial patients and begin to develop a method and framework for evaluating clinical trial patient satisfaction, creating benchmarks, hearing the clinical trial patient voice, and making local improvements in response.

\section{Methods}

\section{Setting}

The MultiCare Institute for Research and Innovation (MIRI) is the largest medical research organization in the South Puget Sound region of Washington State. Embedded within the MultiCare community Hospital System (MHS), MIRI conducts research into more than ten therapeutic areas at 19 clinical sites. Federally funded oncology clinical trials had been conducted at MultiCare for nearly three decades prior to the launch of MIRI in its current form, and that work continues today with MIRI serving as primary recipient for the Northwest National Cancer Institute Community Oncology Research Program.

With MIRI's establishment came a formal expansion of research endeavors into additional therapeutic areas and the development of an investigator-initiated research program. In 2014, MIRI had over 800 patients active in 87 industry-sponsored or 65 federally funded clinical trials. Fifty-three MHS employed physicians acted as principal or sub-investigators on these trials. Although MIRI has its own staff of over 20 clinical research coordinators/assistants, patients are seen in MultiCare facilities and our staff members are all MultiCare employees. Further blurring the distinction between MultiCare and MIRI, many trials include visits during which both standard-of-care and research specific procedures occur. Research patients are unlikely to make a distinction between the research and routine care aspects of their visits, thus strengthening the association between the clinical trial patient experience and patient perception of the MultiCare system as a resource for nonresearch-related medical care.

\section{Survey development}

To create a patient satisfaction survey tailored to clinical trial patients we reviewed the literature $[8,15-18]$, the standardized outpatient survey distributed by a third-party vendor with which our organization contracts, surveys developed by two internal departments (Home Health and Hospice and Sleep Disorders Center), and additional patient satisfaction surveys that were freely available online. Several common areas of inquiry emerged from these reviews including satisfaction with scheduling, facility, staff/ providers, overall satisfaction, and likelihood to return for further care or to recommend the facility to family/friends. We also conducted two brainstorming sessions, first as the MIRI leadership team and subsequently with our institution's Research Oversight Committee, to identify areas of inquiry specific to the clinical trial patient experience. As the survey was to be used as part of our quality improvement process, we did not apply for Institutional Review Board approval prior to mailing it to patients. However, the MultiCare Institutional Review Board granted exemption for use of the results from this anonymous survey for manuscript preparation in April 2015 under protocol number EX-95, and return of a completed survey was considered implied consent.

Development of this survey held the dual purpose of first evaluating the feasibility of distributing and analyzing 
results from a clinical trial participant patient satisfaction survey, and subsequently establishing an internal baseline for use in ongoing assessment of the experience of this unique patient population until a national benchmark becomes available. The author (BMP) developed an initial draft of the survey which was then refined with input from the four-member MIRI leadership team (PJA, AR, RP, and SR) responsible for oversight of all research operations and staff. The penultimate version of the survey was then vetted by MultiCare's Research Oversight Committee which is comprised of 18 physician leaders and high-level administrators and executives within the MultiCare system. The leadership team, in concert with the Research Oversight Committee, identified patients who had (1) consented to a trial in the past year or (2) completed a trial in the past year to which they had consented more than 1 year prior as target survey recipients. The goal of this stratification was to be able to capture a range of recent patient experiences while simultaneously identifying disparities in the perspectives of patients who were at the beginning or end of their research participation. Due to resource limitations, no follow-up phone calls or emails were made after mailing the survey.

The final instrument (see Additional file 1) included seven questions related to the study (how they learned of the study, medical condition, number of visits, etc.), 27 questions on a 5-point Likert scale (Strongly Agree, Agree, Neutral, Disagree, Strongly Disagree), two ranking questions, three free-text response options, and four demographic questions (sex, age, ethnicity, and zip code) for a total of 45 questions on three pages. The instrument also included a preface statement requesting feedback, instructing the patient to return the questionnaire in the enclosed postage-paid envelope, and informing the patient that all responses would be anonymous and kept confidential. As this was the initial foray into assessment of the clinical trial patient experience, the decision was made to allow the survey to be longer than others that we reviewed and to include several potentially related questions. We proceeded with the expectation that we would reduce the length of the survey in the following year after reflecting upon the survey administration experience, analyzing results, and identifying questions that most effectively captured the data of interest.

\section{Analyses}

Likert scale questions were evaluated using both a "top box" score (the percentage of respondents who selected Strongly Agree for each question) and a Six Sigma percentile rank procedure [19]. The percentile rank procedure compares each question's mean to a benchmark value of 4 (Agree) [20], incorporating the variability of the responses by normalizing the data and finding a percentile rank based on the standard normal distribution. Correlation analysis was used to evaluate associations between intended future use of our health care system for routine care and the 26 other Likert measures of patient satisfaction. Pairwise correlations between all Likert scale items were also reviewed to identify redundant questions and unexpected associations. Free-text responses were not formally analyzed, but were reviewed from a quality improvement perspective as anecdotal feedback. Surveys with missing data were excluded from analyses at the question level only. Holm-Bonferroni adjusted $p$ values were calculated to account for the burden of multiple testing. All analyses were performed in the R statistical computing environment [21].

\section{Results}

In June 2014 the survey was mailed to 226 patients identified as having (1) consented to a study in the past year $(N=125,55 \%)$ or (2) completed a trial in the past year to which they had consented more than 1 year prior $(N=101,45 \%)$. Three surveys were returned with no forwarding address and one patient died prior to receiving the survey, so the survey was believed to have been received by 222 patients $\left(N_{\text {consented }}=122, N_{\text {completed }}=\right.$ 100). Ninety surveys (41 \%) were returned between June and November 2014, 81 of which (90\%) were received within 6 weeks of the mailing. Missing data were present in six $(6.6 \%)$ surveys: four $(4.4 \%)$ were missing one data point, one $(1.1 \%)$ was missing two data points, and one $(1.1 \%)$ patient completed only the first page of the survey. A significantly higher proportion of the patients in the consented group returned the survey compared to the patients who had completed a study (50\% versus $29 \%, p=0.002$ ). Forty-four respondents $(48.8 \%)$ were female, 63 (70.0 \%) were aged 55 or older and $73(81.1 \%)$ self-identified as Caucasian. Fifty-two respondents $(57.7 \%)$ reported a home zip code within 15 miles of MIRI's main research facility (Tables 1 and 2). Respondent distribution by therapeutic area is presented in Table 3 .

The highest positive responses were related to patientstaff interactions, with a percentile rank of 90 or higher for staff friendliness (96.8th percentile), respect for patients (94.9th percentile), time spent with patients (94.9th percentile), explanation of role in the study (90.8th percentile), and answering questions fully (90.3th percentile; Table 4). Facility cleanliness and environment were wellrated (86.7th percentile and 81.3th percentile) but parking and convenience of location less so (68.6th percentile and 61.7th percentile). Although the rankings for satisfaction with care and the likelihood of returning to the provider were at the 83rd percentile, overall questions related to enrolling in future trials, enjoying visits, and believing that medical care was enhanced by the study ranked below the 62nd percentile. Fifty-one point one percent of patients 
Table 1 Demographics and background

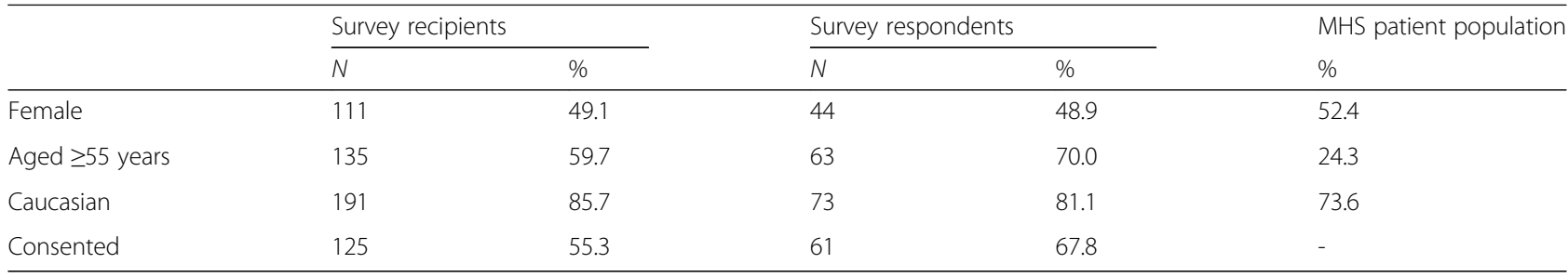

MHS MultiCare Health System

strongly agreed that they would seek future care in our hospital system (64th percentile).

Six items were significantly correlated with participant intention to seek future medical care at a MultiCare facility. All of these correlations were positive and moderate (0.36-0.54, Table 5), and four relate to the relationship with staff. Patient intent to return to the health care provider for routine care was most highly correlated with this item $(r=0.54, p<0.0001)$. Intent to encourage others to participate in studies $(r=0.34, p=0.0013)$ and enjoyment of study visits $(r=0.33, p=0.0016)$, although statistically significant, had Pearson correlation values that are generally considered weak. Evaluation of all pairwise correlations showed strong correlations between perception of a pleasant environment and ease of visit scheduling $(r=0.85)$, trust in research staff $(r=0.77)$, confidence in skills/knowledge of staff $(r=0.72)$, and perception of rapid and full response to concerns $(r=0.70$, all $p<0.0001)$.

Sixty-seven respondents $(74.4 \%)$ cited contribution to medical science and potentially helping others with similar conditions as a primary motivation for volunteering for their research study. An additional 61 respondents (67.8 \%) indicated that a primary motivation was the hope that their own medical condition would be improved. Financial incentives were least cited $(N=16,17.8 \%)$ as a primary motivation for participation (Table 6).

\section{Discussion}

This preliminary evaluation of research patient satisfaction demonstrates that querying this special patient population is feasible and yields valuable insight. Our response rate of $41 \%$ is nearly 2.5 times that of the $17 \%$ response rate to our hospital system's vendor-based outpatient services survey. This may be reflective of a high

Table 2 Additional respondent characteristics

\begin{tabular}{lll}
\hline & Number & Percent \\
\hline Residence zip code <15 miles from main MIRI facility & 59 & 65.6 \\
Received care at MHS prior to study enrollment & 76 & 84.4 \\
Doctor recommended the study during a visit & 67 & 74.4 \\
Made $\geq 4$ visits for the study & 62 & 68.9 \\
\hline
\end{tabular}

MHS MultiCare Health System, MIRI MultiCare Institute for Research and Innovation level of engagement of patients who participate in clinical trials, or suggestive of a heightened willingness of this population to share their perspective; regardless, this response rate underscores the need for the clinical trial patient voice to be heard by the research community. Patients who had consented to a study in the past year were more likely to reply to the survey than patients who had completed a study in the past year. This could be due to a feeling of the experience being more "fresh" as study enrollment was concurrent to survey receipt. Motivations of our research patients were similar to those described in the literature [11, 12, 15, 16, 22], with altruism acting as the primary motivating factor in the decision to enroll on a trial. Our staff was highly rated by participants and free-text responses reflected satisfaction with the staff. However, in the absence of national benchmarks and systematic polling of clinical trial patients immediately following a visit it is not possible to execute a true assessment of the quality of patient-staff interactions in our research institute, as these seemingly "high" scores are not situated in a broader patient experience context and it is unknown if patients who had a negative clinical trial experience were prone to respond to this one-time mailing that may have occurred up to a year after their visit.

The observed correlations between an intent to return to our system for care and several variables were weak to moderate (0.33-0.54) and the associations warrant further explanation before basing process or organization changes on them. Fifty-four percent of the patients who indicated intent to return to MHS for routine care reported a zip code within 15 miles of our main research facility, but with system medical facilities throughout the South Puget Sound region it is difficult to ascertain the extent to which distance influenced this response. An unanticipated finding was the set of strong correlations between perception of a pleasant environment and modifiable aspects of the study, such as scheduling, trust/confidence in the staff, and satisfactory response by staff to patient concerns.

Local directions for future work include revision of the clinical trial patient survey to align with the vendorbased outpatient survey used by our institution. We believe that this will enable us to locate our own patient satisfaction profile within the broader context of patient 
Table 3 Survey respondent therapeutic area of study

\begin{tabular}{|c|c|c|c|c|c|}
\hline \multirow[b]{2}{*}{ Therapeutic area } & \multicolumn{2}{|c|}{ Survey recipients } & \multicolumn{2}{|c|}{ Survey respondents } & \multirow{2}{*}{$\begin{array}{l}\% \text { responded within } \\
\text { therapeutic area } \\
\%\end{array}$} \\
\hline & N & $\%$ & $N$ & $\%$ & \\
\hline Cardiovascular & 40 & 17.7 & 18 & 20.0 & 45.0 \\
\hline Endocrinology & 24 & 10.6 & 12 & 13.3 & 50.0 \\
\hline Internal medicine & 3 & 1.3 & 2 & 2.2 & 66.7 \\
\hline Neurology & 43 & 19.0 & 16 & 17.8 & 37.2 \\
\hline Oncology & 26 & 11.5 & 10 & 11.1 & 38.5 \\
\hline Pediatrics & 22 & 9.7 & 2 & 2.2 & 9.1 \\
\hline Pulmonology & 62 & 27.4 & 25 & 27.8 & 40.3 \\
\hline Stroke & 6 & 2.7 & 3 & 3.3 & 50.0 \\
\hline Not reported & - & - & 2 & 2.2 & - \\
\hline
\end{tabular}

Table 4 Survey questions and results

\begin{tabular}{|c|c|c|c|c|c|c|}
\hline Question & $\mathrm{NA}$ & $\%$ strongly agree & Percentile rank & Mean & $S D$ & $Z$ score \\
\hline Friendly staff & 1 & 80.0 & 96.8 & 4.80 & 0.43 & 1.85 \\
\hline Respectful staff & 1 & 75.6 & 94.9 & 4.75 & 0.46 & 1.64 \\
\hline Adequate time spent & 1 & 72.2 & 94.9 & 4.73 & 0.45 & 1.64 \\
\hline Staff explained role & 1 & 66.7 & 90.8 & 4.66 & 0.50 & 1.33 \\
\hline Staff answered questions & 1 & 65.6 & 90.3 & 4.65 & 0.50 & 1.30 \\
\hline Staff explained procedures & 1 & 64.4 & 88.2 & 4.63 & 0.53 & 1.19 \\
\hline Fully informed of risks/benefits & 1 & 63.3 & 87.7 & 4.62 & 0.53 & 1.16 \\
\hline Staff protected privacy & 1 & 65.6 & 87.3 & 4.63 & 0.55 & 1.14 \\
\hline Trusted staff & 1 & 64.4 & 86.8 & 4.62 & 0.55 & 1.12 \\
\hline Clean facility & 0 & 66.7 & 86.7 & 4.63 & 0.57 & 1.11 \\
\hline Staff were prepared & 1 & 71.1 & 86.5 & 4.66 & 0.60 & 1.10 \\
\hline Reasonable wait time & 0 & 61.1 & 86.3 & 4.59 & 0.54 & 1.09 \\
\hline Confident in staff skills/knowledge & 1 & 63.3 & 86.2 & 4.61 & 0.56 & 1.09 \\
\hline Staff responded to concerns & 1 & 65.6 & 84.4 & 4.62 & 0.61 & 1.01 \\
\hline Extremely satisfied with care & 1 & 64.4 & 83.3 & 4.60 & 0.62 & 0.97 \\
\hline Would return to provider for care & 1 & 66.7 & 83.0 & 4.62 & 0.65 & 0.95 \\
\hline Visit times were convenient & 0 & 61.1 & 81.5 & 4.56 & 0.62 & 0.90 \\
\hline Environment was pleasant & 0 & 63.3 & 81.3 & 4.57 & 0.64 & 0.89 \\
\hline Scheduling visits was easy & 0 & 63.3 & 78.5 & 4.54 & 0.69 & 0.79 \\
\hline Received reminders for visits & 4 & 57.8 & 71.7 & 4.45 & 0.79 & 0.57 \\
\hline Parking was easy & 1 & 52.2 & 68.6 & 4.38 & 0.79 & 0.48 \\
\hline Will seek future care at MHS & 1 & 51.1 & 64.5 & 4.33 & 0.88 & 0.37 \\
\hline Convenient location & 1 & 45.6 & 61.7 & 4.25 & 0.83 & 0.30 \\
\hline Would do another study & 1 & 52.2 & 61.3 & 4.27 & 0.94 & 0.29 \\
\hline Enjoyed visits & 1 & 38.9 & 59.1 & 4.18 & 0.78 & 0.23 \\
\hline Will encourage others to do studies & 1 & 35.6 & 52.1 & 4.04 & 0.84 & 0.05 \\
\hline Medical care enhanced by study & 1 & 40.0 & 50.0 & 4.00 & 1.00 & 0.00 \\
\hline
\end{tabular}


Table 5 Significant correlations

\begin{tabular}{|c|c|c|c|}
\hline Likert scale items & Correlation & $P$ value & $95 \% \mathrm{Cl}$ \\
\hline \multicolumn{4}{|l|}{ I will seek future medical care at a MultiCare facility: } \\
\hline I would return to this health care provider for routine care & 0.54 & $<0.001$ & $0.38-0.67$ \\
\hline Staff members treated me with respect & 0.40 & 0.003 & $0.21-0.56$ \\
\hline I will encourage family and friends to participate in studies & 0.34 & 0.003 & $0.14-0.51$ \\
\hline Staff responded quickly and fully to my concerns & 0.38 & 0.005 & $0.19-0.55$ \\
\hline I trusted the research staff members & 0.38 & 0.006 & $0.18-0.54$ \\
\hline Staff members were friendly & 0.36 & 0.013 & $0.16-0.53$ \\
\hline \multicolumn{4}{|l|}{ The environment was pleasant: } \\
\hline Scheduling my visits was easy & 0.85 & $<0.001$ & $0.78-0.90$ \\
\hline I trusted the research staff members & 0.77 & $<0.001$ & $0.67-0.85$ \\
\hline I was confident in the skills and knowledge of the staff & 0.72 & $<0.001$ & $0.61-0.81$ \\
\hline Staff responded quickly and fully to my concerns & 0.70 & $<0.001$ & $0.58-0.79$ \\
\hline
\end{tabular}

satisfaction in our organization until national benchmarking data become available through widespread evaluation of the clinical trial patient experience. It has been shown that patient satisfaction is best assessed using surveys targeted to a specific visit rather than attempting to evaluate a general perception of an experience [6] and, moving forward, we aim to administer the survey as patient visits occur and to target evaluation of specific visits. Indeed, after reviewing responses for the survey discussed here, we revised our instrument and now distribute a two-page instrument with 27 Likert scale questions and seven demographic questions. This instrument is sent to patients immediately following a consent, annual, or end of study visit. We intend to report results from this instrument in a future publication.

A substantial limitation of this work is the lack of national benchmark data for the United States. We hope that this preliminary effort to evaluate patient satisfaction of clinical trial participants will act as a call to action in the broader research community to begin evaluation of the research patient experience and the impact of research on the patient perception and utilization of health care systems, ultimately leading to a nationally standardized system of evaluation. This is of special

Table 6 Motivations for volunteering

\begin{tabular}{lll}
\hline Motivation & Number & Percent \\
\hline $\begin{array}{l}\text { To contribute important information to } \\
\text { medical science }\end{array}$ & 67 & 74.4 \\
$\begin{array}{l}\text { To potentially help other people with } \\
\text { similar conditions }\end{array}$ & 67 & 74.4 \\
$\begin{array}{l}\text { I hoped that the research study would } \\
\text { improve my medical condition }\end{array}$ & 61 & 67.8 \\
$\begin{array}{l}\text { To gain insights into my own health } \\
\text { To benefit from the additional medical } \\
\text { attention and testing that the study provided } \\
\text { Because of the financial incentives of the study }\end{array}$ & 42 & 46.7 \\
\hline
\end{tabular}

importance as, with any survey, the findings in this study are based on a voluntary response sample and without repeated and diverse sampling, the generalizability of the results is diminished. Such repeated sampling is also crucial if we are to identify areas of greatest importance to the patients themselves, both to improve practices nationwide and to develop more concise instruments for receiving feedback from these important volunteers. Future inquiry into research patient satisfaction would benefit greatly from a mixed-methods approach, including analysis of structured interviews, to develop a more nuanced description of the research patient experience. Ultimately, research will also be required to determine the differences in how patient satisfaction is measured and what is of greatest import for patients engaging in clinical research in countries with national health care compared to that of research patients in the United States.

We hope, with this work, to be able to share our lessons learned with other members of the research community and proceed in collaboration with other research organizations to develop and deploy a standardized survey, thereby beginning the important work of creating a foundational understanding of clinical trial patient satisfaction. Without such a system we are at risk of misunderstanding and effectively responding to the patient voice. We now have an opportunity to explore another realm of inquiry into clinical trial research and to validate the generous contributions made by patients who put themselves and their families at the forefront of medical breakthroughs.

\section{Conclusions}

Satisfaction of patient volunteers in clinical trials is grossly understudied. In an era of increasing focus on the patient experience, this important subset of patients receiving research-related health care services needs to be queried to obtain a better understanding of how we 
can best serve these patients and how their experience in research impacts their use of health care systems for nonresearch-related care. Some similarities to nonresearch patient satisfaction seem to be present, such as a strong influence of interactions with care providers, but a national movement is needed among organizations conducting clinical trials in order to develop benchmarks and create and understand the connections for these patients between research-related and nonresearch-related care.

\section{Additional file}

Additional file 1: Survey instrument.pdf (copy of instrument used to collect data reported in this manuscript). (PDF 335 kb)

\section{Abbreviations}

HCAHPS: Hospital Consumer Assessment of Healthcare Providers and Systems; MHS: MultiCare Health System; MIRI: MultiCare Institute for Research and Innovation

\section{Authors' contributions}

BMP developed and deployed the survey, analyzed the survey results, and drafted the manuscript. PJA presented the penultimate survey to the Research Oversight Committee for feedback. SR, AR, RP, and PJA contributed substantially to survey development and deployment. PJA and AR critically reviewed the manuscript and all authors read and approved the final manuscript.

\section{Competing interests}

The authors declare that they have no competing interests.

Received: 13 November 2015 Accepted: 23 September 2016 Published online: 06 October 2016

\section{References}

1. Centers for Medicare and Medicaid Services. Hospital Consumer Assessment of Healthcare Providers and Systems. 2016. Available from: http://www.hcahpsonline.org. Accessed date 11 Jul 2016

2. Centers for Medicare and Medicaid Services. Hospital value-based purchasing program frequently asked questions. Available from: https://www.cms.gov/Outreach-and-Education/Medicare-LearningNetwork-MLN/MLNProducts/downloads/Hospital_VBPurchasing_Fact_ Sheet_ICN907664.pdf. Accessed 30 Jul 2015.

3. Jha AK, et al. Patients' perception of hospital care in the United States. N Engl J Med. 2008;359(18):1921-31.

4. Glickman SW, et al. Patient satisfaction and its relationship with clinical quality and inpatient mortality in acute myocardial infarction. Circ Cardiovasc Qual Outcomes. 2010;3(2):188-95.

5. Otani K, et al. Patient satisfaction: focusing on "excellent". J Healthc Manag. 2009;54(2):93-102. discussion 102-3.

6. Manary MP, et al. The patient experience and health outcomes. N Engl J Med. 2013;368(3):201-3.

7. Elliott MN, et al. Accelerating improvement and narrowing gaps: trends in patients' experiences with hospital care reflected in HCAHPS public reporting. Health Serv Res. 2015;50(6):1850-67. doi:10.1111/1475-6773.12305. Epub 2015 Apr 8.

8. Verheggen FW, et al. Patient satisfaction with clinical trial participation. Int J Qual Health Care. 1998;10(4):319-30.

9. Chu SH, et al. The views of patients and healthy volunteers on participation in clinical trials: an exploratory survey study. Contemp Clin Trials. 2012;33(4): 611-9.

10. Jenkins $\mathrm{V}$, et al. The attitudes of 1066 patients with cancer towards participation in randomised clinical trials. Br J Cancer. 2010;103(12):1801-7.

11. Jones JM, et al. Identifying motivations and barriers to patient participation in clinical trials. J Cancer Educ. 2006;21(4):237-42.

12. Bevan $\mathrm{EG}$, et al. Patients' attitudes to participation in clinical trials. Br J Clin Pharmacol. 1993;35(2):204-7.
13. Planner C. Measuring patients' experience of clinical trials: results of an exploratory review and stakeholder workshop, in 3rd International Clinical Trials Methodology Conference. Trials: Glasgow. 2015;16(Suppl 2):P113.

14. Bray C. Surveying clinical trial participant satisfaction, in 3rd International Clinical Trials Methodology Conference. Trials: Glasgow. 2015;16(Suppl 2):P45.

15. Henzlova MJ, et al. Patient perception of a long-term clinical trial: experience using a close-out questionnaire in the Studies of Left Ventricular Dysfunction (SOLVD) trial. SOLVD Close-out Working Group. Control Clin Trials. 1994:15(4):284-93.

16. Schron EB, Wassertheil-Smoller S, Pressel S. Clinical trial participant satisfaction: survey of SHEP enrollees. SHEP Cooperative Research Group. Systolic Hypertension in the Elderly Program. J Am Geriatr Soc. 1997;45(8):934-8.

17. Shirley ED, Sanders JO. Patient satisfaction: implications and predictors of success. J Bone Joint Surg. 2013;95(10):e69 1-4.

18. Tangrea JA, Adrianza ME, Helsel WE. Patients' perceptions on participation in a cancer chemoprevention trial. Cancer Epidemiol Biomarkers Prev. 1992; 1(4):325-30.

19. Sauro J. How to interpret survey responses: 5 techniques. 2011. Available from: http://www.measuringu.com/blog/interpret-responses.php. Accessed 14 Apr 2015.

20. Nielsen J, Levy J. Measuring usability: preference vs. performance. Commun ACM. 1994:37(4):66-75

21. R Core Team. R: a language and environment for statistical computing. Vienna: R Foundation for Statistical Computing; 2013. URL http://www.Rproject.org/. Accessed 29 Jan 2014.

22. Mattson ME, Curb JD, McArdle R. Participation in a clinical trial: the patients' point of view. Control Clin Trials. 1985;6(2):156-67.
Submit your next manuscript to BioMed Central and we will help you at every step:

- We accept pre-submission inquiries

- Our selector tool helps you to find the most relevant journal

- We provide round the clock customer support

- Convenient online submission

- Thorough peer review

- Inclusion in PubMed and all major indexing services

- Maximum visibility for your research

Submit your manuscript at www.biomedcentral.com/submit
) Biomed Central 